\title{
Elimination des bactéries et des pyrogènes par ultrafiltration
}

par

\author{
A. ARMAND
}

L'une des premières applications industrielles du matériel membranaire a été l'emploi de systèmes, l'ultrafiltration et l'osmose inverse, dans la réalisation d'une partie du procédé permettant d'obtenir l'eau de très haute qualité indispensable au lavage des circuits imprimés et autres composants électroniques. A l'heure actuelle, l'ultrafiltration ou l'osmose inverse constituent une phase de ce type de procédé. En ce qui concerne le domaine pharmaceutique et biochimique, bien que le matériel utilisé soit essentiellement le même, il existe cependant une différence capitale. Celle-ci réside dans le fait que les équipements électroniques et, d'une manière générale, les équipements industriels ne soient pas conçus en fonction des normes d'hygiène, alors que ceux destinés aux activités pharmaceutiques et biochimiques doivent l'être absolument. La raison de cette différence tient, bien entendu, à l'usage que l'on fait de l'eau produite.

La nécessité de s'assurer de l'absence de pyrogènes dans l'eau ou les solutions peut constituer un problème important pour les fabricants de produits pharmaceutiques et les laboratoires de clinique et de recherche. Bien que toute substance engendrant une élévation de température lors de son injection entre dans la définition des pyrogènes, la plupart d'entre eux sont des lipopolysaccharides (LPS) issus des parois des cellules bactériennes.

Les pyrogènes ne peuvent pas être éliminés par passage à l'autoclave ni par filtration à l'aide d'une membrane microporeuse. Les procédés les plus largement utilisés jusqu'à maintenant sont la distillation, l'osmose inverse (OI) et l'absorption par l'amiante ou autres moyens. Les inconvénients de ces méthodes résident dans le coût élevé de l'investissement et de l'exploitation de la distillation, dans la difficulté que présente la stérilisation des systèmes OI et dans

$\alpha$ Alfa-Laval Industrie, Z.I. Le Chêne Sorcier, C.D. 161, B.P. 56 - 78340 Les Clayes-sous-Bois. 
le rendement irrégulier des absorbants auxquels il convient d'ajouter le fait que l'exposition à l'amiante est peu souhaitable. En outre, ni la distillation ni l'OI ne peuvent être employées pour dépyrogéniser les solutions pour injection parentérale appartenant aux catégories à faible poids moléculaire.

\section{TERMINOLOGIE}

Polysaccharides : catégorie d'hydrates de carbone dont les molécules contiennent des chaînes monosaccharidiques : comprend l'amidon, l'inuline et la cellulose. Formule générale : $\left(\mathrm{C}_{6} \mathrm{H}_{10} \mathrm{O}_{5}\right)_{\mathrm{n}}$.

Lipides : vaste groupe de composés organiques comprenant les esters ou les acides gras (lipides simples tels que les graisses et les cires) ou les substances étroitement apparentées (lipides complexes, tels que les phospholipides) : habituellement insolubles dans l'eau mais solubles dans l'alcool et autres solvants organiques. Ils constituent des matériaux structurels importants dans les organismes vitaux.

Lipopolysaccharide /LPS) : polysaccharide auquel est liée une molécule lipidique. Composant de la membrane externe des bactéries.

Pyrogènes : groupe de substances, comprenant les toxines, engendrant une élévation de la température corporelle de l'homme et de l'animal, c'est-à-dire qui provoquent une fièvre. La plupart des pyrogènes sont des lipopolysaccharides (LPS) provenant de la membrane externe des bactéries. La taille de ces endotoxines peut s'échelonner entre les sous-unités dont le poids moléculaire (PM) est d'environ 20000 et les agrégats dont le diamètre est d'environ $0,1 \mu \mathrm{m}$. Le seuil de détectabilité est compris entre 0,01 et $0,1 \mathrm{ng} / \mathrm{ml}$.

\section{QU'EST-CE QUE L'EAU?}

Chacun sait ce qu'est l'eau, mais le sait-il vraiment? Si on donne à goûter à quelques personnes de l'eau hautement purifiée la réaction générale est la suivante : c'est " plat ", " ça n’a pas de goût », c'est " insipide », " il manque quelque chose ». La raison de cette réaction, c'est que ce que nous buvons et utilisons quotidiennement n'est pas de l'eau pure. C'est de l'eau assortie de sels inorganiques et de gaz dissous, et, probablement, de quelques composés organiques. En ce qui concerne l'eau potable, ces derniers ne seront pas considérés comme contaminants sauf si le niveau total de solides dissous (TSD) dépassait le chiffre de $700 \mathrm{ppm}$. Les recommandations de I'O.M.S. portent sur un TSD maximum de $500 \mathrm{ppm}$, mais dans de 
nombreux pays les gens sont habitués à boire des eaux présentant des niveaux de TSD plus élevés, jusqu'à 1500 ppm au Moyen-Orient. En fait, de nombreuses eaux « minérales » présentent des valeurs de TSD avoisinantes, ou supérieures, ainsi que des taux élevés ou très élevés de gaz dissous. Selon le pays, l'eau peut également contenir davantage de composés organiques, quelques macroparticules, des colloïdes, et même des bactéries et des virus.

Les composés organiques proviennent à l'origine des organismes vivants, les plus dangereux étant issus des bactéries pathogènes. Selon les normes classiques, on considère que l'ensemble de ces contaminants tels que les contaminants présents dans l'eau potable, c'est-à-dire l'eau de boisson ou l'eau pour la consommation humaine, sont dans le meilleur des cas indésirables, ou dangereux dans le pire des cas.

En ce qui concerne l'industrie pharmaceutique, l'eau potable est hautement contaminée et représente la catégorie inférieure des différentes qualités d'eau utilisées.

\section{TESTS METTANT EN EVIDENCE LES PYROGENES}

Jusqu'à ces dernières années, il n'existait qu'un seul test pour mettre en évidence les pyrogènes, qui constitue en réalité autant un test qu'une définition des pyrogènes. Il s'agit du test reposant sur la réaction fébrile des lapins. Dans des conditions réglementées une substance est définie comme contenant des pyrogènes si elle engendre chez le lapin une élévation de température supérieure à un niveau donné (c'est-à-dire, de la fièvre) (soit : " pyro », température élevée et " gène " qui produit).

La valeur de ce test réside dans le fait que l'on a la quasicertitude de détecter les pyrogènes. L'inconvénient c'est que ce qui provoque une élévation de la température, ce n'est pas seulement un élément dangereux comme les lipopolysaccharides provenant de la membrane extérieure des bactéries, mais ce sont aussi les sels inorganiques ou l'environnement, et/ou la méthode de manipulation des lapins.

Il y a quelques années un nouveau test appelé le test LAL (Lysat d'Amoebocytes du limulus) ou encore "limulus test" N. du T.), repose sur les propriétés de gélification et/ou de turbidité d'un lysat préparé à partir des amoebocytes circulants des limules américains et japonais (Limulus polyphemus et Limulus tachypleus). D'une part, il s'agit là d'un test plus sensible que le test des lapins (100 à 1000 fois) mais, par contre, il ne peut détecter que les pyrogènes provenant des bactéries à Gram négatif, et un débat important a eu lieu aux Etats-Unis sur la question de savoir si le degré de 
sensibilité est, en fait, trop élevé, de sorte qu'on obtient un trop grand nombre de faux résultats positifs.

Il est très peu probable que le test du lapin soit abandonné. Il paraît plus vraisemblable qu'il y aura un choix de tests pour la majorité des applications avec une spécificité de l'un ou l'autre des tests pour un nombre plus restreint de situations spécifiques.

\section{ULTRAFILTRATION - GENERALITES}

On a recours à l'ultrafiltration pour éliminer de l'eau et des solutés pour administration parentérale les micro-organismes, les bactéries, les spores de micro-organismes, les colloïdes, les toxines et les pyrogènes LPS.

Pour les applications de l'eau l'alimentation en eau de l'équipement d'ultrafiltration est souvent déionisée. L'eau produite par l'appareil d'ultrafiltration, le filtrat, est utilisée de diverses manières, y compris dans le lavage et le rinçage finals des récipients pour les produits pharmaceutiques et les cosmétiques tels que les bouteilles et les fioles et dans la préparation des produits pharmaceutiques et cosmétiques non-éthiques (c'est-à-dire, délivrés sans ordonnance), par exemple les onguents et les crèmes de soin pour la peau.

Il faut souligner que l'UF ne convient pas pour la production d' " eau pour injection », c'est-à-dire l'eau utilisée pour la préparation des médicaments ou autres substances injectées dans l'organisme. (A une exception près, la seule méthode autorisée pour la préparation de l' " eau pour injection ", c'est la distillation. Ce sont les Etats-Unis qui constituent l'exception, où une osmose inverse à double phase est tolérée). On ne devra envisager l'ultrafiltration que pour la production d' "Eau Purifiée ».

On peut utiliser l'UF pour produire des solutés pour administration parentérale bactériologiquement stériles. On doit aborder cas par cas la question de l'utilisation de l'UF pour dépyrogéniser les solutés pour administration parentérale, après en avoir délibéré avec le client, et en tenant compte de la règlementation de la Pharmacopée en vigueur. Il faut d'autre part bien préciser que l'UF n'est pas en mesure d'éliminer entièrement tous les pyrogènes sur la base d'une définition obtenue à partir du test du lapin mais seulement les pyrogènes LPS que l'on rencontre normalement.

\section{ULTRAFILTRATION - DESCRIPTION}

La membrane normalement employée pour la seule élimination des bactéries est la GM80. Etant donné que des débits aussi élevés 
que 170 à $190 \mathrm{l} / \mathrm{m}^{2} \mathrm{~h}$ pour l'eau déionisée, et de 85 à $96 \mathrm{l} / \mathrm{m}^{2} \mathrm{~h}$ pour l'eau de ville sont normaux, avec la GM80 il est possible de concevoir un équipement qui fonctionne sur la base d' " un passage unique ", c'est-à-dire sans recyclage, puisqu'on peut récupérer jusqu'à $95 \%$ de l'eau d'alimentation comme filtrat au cours d'un seul passage à travers l'équipement d'UF. Ceci apparaît sur la figure 1.

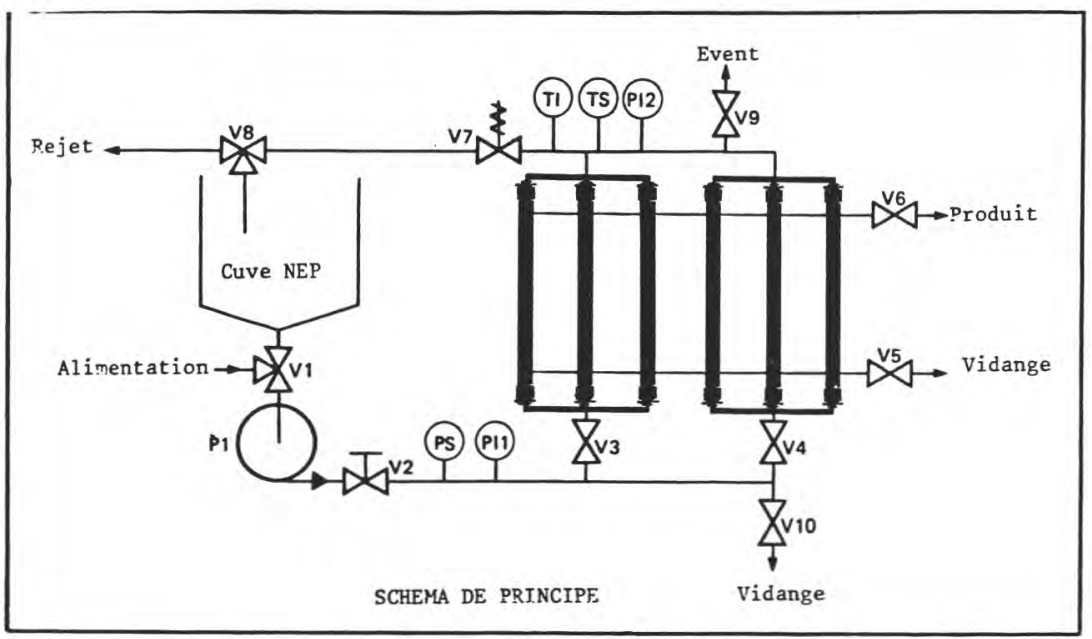

fig. 1

Pour l'élimination des bactéries et des pyrogènes LPS on utilise la membrane PM10. Le rendement du flux est là aussi extrêmement variable selon la source et la qualité de l'eau et il se tiendra vraisemblablement dans la fourchette comprise entre 35 et $70 \mathrm{l} / \mathrm{m}^{2} / \mathrm{h}$. Selon la méthode exacte employée, on peut récupérer jusqu'à environ $50 \%$ de l'eau d'alimentation sous forme de filtrat en un seul passage. Le recyclage pourra être envisagé à des valeurs plus basses.

Quelques données concernant l'élimination des pyrogènes LPS figurent dans les tableaux 1 et 2. Dans le cas du tableau 1 on a ajouté de façon délibérée des taux élevés de pyrogènes, l'expression "Stimulation de la membrane »y fait allusion.

Le tableau 2 donne les chiffres d'élimination pour les pyrogènes LPS et les bactéries à partir de l'eau déionisée sur une longue période. 


\section{$T A B L E A U 1$}

Elimination des Pyrogènes LPS de l'eau du robinet en utilisant une membrane PM 10

\begin{tabular}{r|c|c}
\hline & \multicolumn{2}{|c}{ Pyrogènes LPS $(\mathrm{ng} / \mathrm{ml})-$ test LAL } \\
\cline { 2 - 3 } Jour & Avant ultrafiltration & Après ultrafiltration \\
\cline { 2 - 3 } & & \\
\cline { 2 - 3 } 1 & 300 & $<0050$ \\
2 & 100 & $\leq 0050$ \\
3 & 300 & $\leq 0050$ \\
4 & 10000 & $\leq 0050$ \\
5 & 1000 & $\leq 0050$ \\
10 & 1000 & $<0050$ \\
15 & 1000 & $<050$ \\
\hline
\end{tabular}

TABLEAU 2

Elimination des bactéries et des pyrogènes LPS à partir de l'eau déionisée en uitilisant la membrane PM 10.

On utilise le test LAL pour la détection des pyrogènes.

\begin{tabular}{|c|c|c|c|c|}
\hline \multirow{2}{*}{ Jour } & \multicolumn{2}{|c|}{ Avant ultrafiltration } & \multicolumn{2}{|c|}{ Après ultrafiltration } \\
\hline & $\begin{array}{c}\text { Comptage des } \\
\text { bactéries } / 100 \mathrm{ml}\end{array}$ & $\begin{array}{c}\text { Pyrogènes LPS } \\
\mathrm{ng} / \mathrm{ml}\end{array}$ & $\begin{array}{c}\text { Comptage des } \\
\text { bactéries } / 100 \mathrm{ml}\end{array}$ & $\begin{array}{c}\text { Pyrogènes LPS } \\
\mathrm{ng} / \mathrm{ml}\end{array}$ \\
\hline $\begin{array}{r}1 \\
2 \\
3 \\
12 \\
19 \\
26 \\
40 \\
54 \\
68 \\
82\end{array}$ & $\begin{array}{r}12000 \\
25000 \\
9000 \\
7000 \\
4000 \\
6000 \\
8000 \\
4000 \\
7000 \\
5000\end{array}$ & $\begin{array}{r}3 \\
10 \\
3 \\
1 \\
3 \\
3 \\
1 \\
3 \\
10 \\
3\end{array}$ & $\begin{array}{l}0 \\
3 \\
0 \\
0 \\
0 \\
0 \\
0 \\
0 \\
0 \\
0\end{array}$ & $\begin{array}{lll}< & 0 & 050 \\
< & 0 & 050 \\
< & 0 & 050 \\
< & 0 & 050 \\
< & 0 & 050 \\
< & 0 & 050 \\
< & 0 & 050 \\
< & 0 & 050 \\
< & 0 & 050 \\
< & 0 & 050\end{array}$ \\
\hline
\end{tabular}

Pour l'élimination des bactéries et/ou des pyrogènes LPS des solutés pour administration parentérale le type de membrane et le rendement dépendra du produit particulier traité et du type optimal de membrane nécessaire pour permettre le passage de la molécule du produit. 
Coût et efficacité de l'UF pour la production d'Eau Purifiée.

Le tableau suivant montre la comparaison entre l'ultrafiltration par fibre creuse et les autres méthodes.

\section{TABLEAU 3}

Comparaison de l'UF à d'autres méthodes pour la production d'Eau Purifiée

\begin{tabular}{|c|c|c|c|c|c|}
\hline & $\begin{array}{l}\text { UF par } \\
\text { fibre } \\
\text { creuse }\end{array}$ & $\begin{array}{l}\text { Distil- } \\
\text { lation }\end{array}$ & $\begin{array}{l}\text { Distil- } \\
\text { lation } \\
\text { thermo. } \\
\text { comp. }\end{array}$ & $\begin{array}{l}\text { Osmose } \\
\text { inverse }\end{array}$ & $\begin{array}{l}\text { Filtres } \\
\text { chargés }\end{array}$ \\
\hline Conception sanitaire & Bonne & Bonne & Bonne & Passable & Bonne \\
\hline $\begin{array}{l}\text { Efficacité de l'élimina- } \\
\text { tion }\end{array}$ & $>10^{5}$ & $>10^{5}$ & $>10^{5}$ & $>10^{5}$ & $10^{2}-10^{3}$ \\
\hline $\begin{array}{l}\text { Coût d'exploitation ap- } \\
\text { proximatif } \\
\text { SEL par } 1000 \text { litres }\end{array}$ & $\begin{array}{l}3- \\
4,5\end{array}$ & $\begin{array}{l}55- \\
85\end{array}$ & $11,5-$ & $5,5-$ & $14-5$ \\
\hline $\begin{array}{l}\text { Coût d'investissement } \\
\text { approximatif pour un } \\
\text { système équivalent à } \\
\text { une surface de mem- } \\
\text { brane entre } 5 \text { et } 20 \mathrm{~m}^{2}\end{array}$ & $\mathrm{X}$ & $8 X$ & $18 \mathrm{X}$ & $2,5 X$ & $0,7 \mathrm{X}$ \\
\hline
\end{tabular}

